Das ölige, bei $245^{\circ}$ bis $250^{\circ}$ übergehende Säuregemisch, das in der Kälte nicht erstarrte, wurde in der zur Auflösung eben notwendigen Menge Natronlauge gelöst, und je eine Probe der neutralen Lösung mit einer konzentrierten Strontiumnitratlösung und einer Lithiumchloridlösung versetzt.

In beiden Fällen fielen sofort die schwerlöslichen fettsauren Salze der betreffenden Metalle aus, die abgesaugt, und nach zweimaligem Umkrystallisieren aus heissem Wasser als einheitliche weisse Blättchen erhalten wurden. Bei $105^{\circ}$ im Trockenschrank getrocknet, ergaben die beiden Salze folgende Analysenresultate:

Lithiumsalz. 10,560 mgr Subst. gaben 3,550 mgr Li $\mathrm{Li}_{3} \mathrm{SO}_{4}$

$$
\begin{array}{r}
\mathrm{C}_{9} \mathrm{H}_{17} \mathrm{O}_{2} \mathrm{Li} \quad \text { Ber. Li } 4,23 \% \\
\text { Gef. " } 4,24 \%
\end{array}
$$

Strontiumsalz. $5,780 \mathrm{mgr}$ Subst. gaben 2,66 mgr $\mathrm{SrSO}_{4}$

$7,430 \mathrm{mgr}$ Subst, gaben $5,40 \mathrm{mgr} \mathrm{H}_{2} \mathrm{O}$ und $14,560 \mathrm{mgr} \mathrm{CO}_{2}$

$$
\begin{array}{llll}
\left(\mathrm{C}_{9} \mathrm{I}_{17} \mathrm{O}_{2}\right)_{2} \mathrm{Sr} & \text { Ber. } \mathrm{Sr} 21,80 & \text { C } 53,73 & \text { H } 8,53 \% \\
& \text { Gef. }, 21,97 & \# 53,47 & \\
& & 8,14 \%
\end{array}
$$

Somit ist die isolierte Säure Nonylsäure (Pelargonsäure).

Zürich, Chemisches Laboratorium der Universität.

\title{
Zur Stereochemie des Rutheniumatoms von
}

\section{A. Werner $\uparrow$ und Al. P. Smirnoff $\left.{ }^{1}\right)$.}

(30. IX. 20.)

Komplexe Rutheniumsalze waren schon dem Entdecker dieses Elements, C. Claus bekannt; A. Joly vermehrte später das vorhandene Material beträchtlich und berichtigte einige Irrtümer bezüglich der Zusammensetzung der schon bekannten Verbindungen²).

1) Vergl. Helv. 3, 472, Fussnote 1 (1920).

2) C. Claus, Bl. Ac. Pétersb. 1, 99 (1858); A. Joly, C. R. 107, 994 (1888). 
Er untersuchte unter anderem eine eigenartige Reihe ammoniakhaltiger komplexer Rutheniumsalze, welche schon Claus zum Teil in den Händen gehabt hatte, und erkannte richtig ihre Zusammensetzung, ohne indessen in ihr Wesen eindringen zu können; er hielt ihren Aufbau sogar für völlig verschieden von demjenigen der bekannten Platinammoniaksalze ${ }^{1}$ ).

Vor mehr als zehn Jahren wurde nun von A. Werner gezeigt, dass sowohl diese, als Nitroso-hydroxo-tetrammin-ruthenesalze anzusprechende Reihe:

$$
\left[\begin{array}{ll}
\mathrm{NO} \\
\mathrm{H} O
\end{array}\right.
$$

als auch die ihr verwandten, durch Ersatz der Hydroxylgruppe erhaltenen Nitroso-chloro- bezw. Nitroso-bromo- und Nitroso-aquoreihen ganz ähnliche Gesetzmässigkeiten aufweisen, wie zahlreiche komplexe Reihen des Platins resp. des Kobalts $\left.{ }^{2}\right)$. Dadurch wurde die Gültigkeit der Koordinationszahl sechs für das Rutheniumatom festgestellt und die Grundlage zur Interpretation anderer komplexer Rutheniumverbindungen geschaffen.

Es blieb aber noch die weitere Folgerung aus der Koordinationslehre, nämlich die oktaëdrische Anordnung der sechs Gruppen um das Zentralatom, zu beweisen übrig. Diese Lücke sollte nun ausgefüllt werden, nachdem der analoge Beweis u. a. für drei Elemente der Platingruppe, $\left.\mathrm{Pt}^{3}\right), \mathrm{Ir}^{4}$ ) und $\mathrm{Rh}^{5}$ ), unter Auswertung der durch jene Anordnung unter gewissen Umständen bedingten Molekelasymmetrie gelungen war. Dies erschien umso wichtiger, als das Rutheniumatom in manchen Verbindungen die Neigung zeigt, scheinbar nur fünf Gruppen an sich direkt zu ketten, während in einigen koordinativ gesättigten Komplexen dieses Elements eine der sechs Stellen vorzugsweise durch besondere Gruppen besetzt ist, wie wenn sie den übrigen fünf nicht gleichwertig wäre. So kennt man sehr stabile Reihen:

$$
\begin{aligned}
& \left.\left.\left[\begin{array}{ll}
\mathrm{Cl}_{5} & \mathrm{Ru}
\end{array}\right] \mathrm{R}_{2}{ }^{6}\right) \text { und }\left[\mathrm{NO} \cdot \mathrm{Ru}_{\mathrm{Cl}} \mathrm{I}_{5}\right] \mathrm{R}_{2}{ }^{7}\right) \\
& (\mathrm{R}=\text { ein einwertiges positives Radikai). }
\end{aligned}
$$

\footnotetext{
1) G. Claus, Bl. Ac. Pétersb. 4, 455 (1862); A. Joly, C. R. 108, 1300 (1889).

2) B. 40, 2616 (1907).

3) Vierteljahrsschr. der Naturf. Ges. Zürich, 62, 563 (1917).

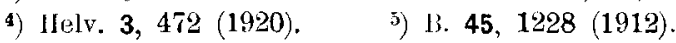

6) I. L. Howe, Am. Soc. 23, 78 If. (1901).

7) A. Joly, C. R. 107, 994 (1888).
} 
Auch hier, wie bei den ähnlichen Iridiumverbindungen, musste erst ein zur Erforschung von räumlichen Verhältnissen guinstiges Material zugänglich gemacht werden, und wiederum erwies es sich - wie dort - als äusserst schwierig, die nötigen Brücken in der Gestalt von Äthylendiaminmolekeln einzuführen, wenn man die Substitution an Halogenokomplexen des drei- bezw. vierwertigen Rutheniums vornahm. Nach vielen misslungenen Versuchen in dieser Richtung fanden wir endlich, dass die oben erwähnte Nitroso-hydroxo-tetrammin-ruthenereihe ihr sämtliches Ammoniak leicht gegen Äthylendiamin austauscht, wobei eine Molekel Diamin an Stelle von zwei Ammoniakmolekeln eintritt. Die so erhaltene neue Reihe ist somit ein Analogon des Mutterkomplexes, wie z. B. die 1,2-Dinitro-diäthylendiamin-kobaltireihe der 1,2-Dinitro-tetrammin-kobaltireihe analog ist; in der Tat enthält der neue Komplex die beiden Gruppen NO und HO noch intakt. Wir werden nun fortan die Salze dieser Reihe als Nitrosohydroxo-diäthylendiamin-ruthenesalze bezeichnen und uns folgender Formel bedienen:

$$
\left[\begin{array}{lll}
\mathrm{NO} & R \mathrm{u} & \mathrm{en} \\
\mathrm{HO} & \mathrm{R}
\end{array}\right] \mathrm{X}_{2}
$$

Die Eigenschaften dieser und der Nitroso-hydroxo-tetramminruthenereihe sind recht ähnlich. Die Salze sind gelb bis orangegelb gefärbt und geben rein gelbe, neutrale Lösungen; sie krystallisieren gut und grösstenteils ohne Krystallwasser. An sich sehr beständig, gehen diese Salze durch Behandlung mit sehr konzentrierten Halogenwasserstoffsäuren in orangefarbene, sauer reagierende Salze der entsprechenden Aquoreihe (I) ïber; bei hoher Temperatur unter Druck wirken diese Säuren weiter, indem las primär gebildete Komplexwasser durch Halogen ersetzt wird, wobei tief braunrote Salze der entsprechenden Chloro- bezw. Bromoreihe (II) entstehen:

$$
\begin{aligned}
& {\left[\begin{array}{lll}
\mathrm{NO} & \mathrm{Ru} & \mathrm{en} \\
\mathrm{HO} & \mathrm{u} & \mathrm{2}
\end{array}\right] \mathrm{x}_{2} \stackrel{+\mathrm{HX}}{\longrightarrow}\left[\begin{array}{lll}
\mathrm{NO} & \mathrm{Ru} & \mathrm{en} \\
\mathrm{H}_{2} \mathrm{O} & &
\end{array}\right] \mathrm{x}_{3} \quad \text { (I) }} \\
& \stackrel{-\mathrm{H}_{2} \mathrm{O}}{\longrightarrow}\left[\begin{array}{lll}
\mathrm{NO} & \mathrm{Ru} & \mathrm{en} \\
\mathrm{X} &
\end{array}\right] \mathrm{x}_{2}
\end{aligned}
$$

Ob den beiden Gruppen NO und HO die Stellung 1,2 (cis) oder 1,6 (trans) zukommt, lässt sich vorläufig nicht entscheiden, 
weil man vor allem die von der Theorie vorausgesehenen Stellungsisomeren der Nitroso-hydroxo-diäthylendiamin-ruthene- bezw. Nitroso-hydroxo-tetrammin-ruthenereihen nicht kennt. Da aber Salze der beiden Reihen sehr ähnliche Lichtabsorption zeigen und fast alle wasserfrei krystallisieren, lässt sich die gleiche Struktur der beiden Komplexe mit grosser Wahrscheinlichkeit annehmen.

Die Anwesenheit von zwei Brücken im neuen Komplex ermöglicht indessen einen weiteren Einblick in seine Struktur, denn die Theorie sieht bekanntlich nur für die 1,2-Diacido-diäthylendiamin-metallkomplexe zwei optisch-aktive Formen voraus. Es sollte daher die Spaltung der neuen Reihe mittelst der so oft erprobten Salzbildungsmethode versucht werden; die Spaltbarkeit des Komplexes würde sowohl für seine oktaëdrische Anordnung wie für die 1,2-Stellung der beiden negativen Gruppen zugleich beweisend sein.

Fünf verschiedene optisch-aktive Säuren wurden nacheinander als Spaltungsmittel verwendet, und zwar: d-( $\alpha$-Brom $)-\pi$ camphersulfonsäure, $d$ - $\alpha$-Camphersulfonäure, d-Camphersäure, d-Nitrocampher und d-Weinsäure - alle ohne den gewünschten Erfolg (s. exp. Teil). Dieses Ergebnis lässt - immerhin mit Vorbehalt - auf 1,6-(trans-) Stellung der Gruppen NO und HO schliessen.

\section{Experimenteller Teil.}

Darstellung des Ausgangsmaterials: Kalium-nitrosopentachloro-rutheneat.

$$
\left[\mathrm{NO} \mathrm{Ru} \mathrm{Cl} \mathrm{S}_{5}\right] \mathrm{K}_{\mathbf{2}}{ }^{1} \text { ) }
$$

50 gr käufliches Ruthenichlorid $\mathrm{RuCl}_{3}$ wurden in einer Porzellanschale auf dem Sandbade mit $500 \mathrm{~cm}^{3}$ konzentrierter Salpetersäure bis zur Syrupdicke eingedampft. Hierauf wurden $500 \mathrm{~cm}^{3}$ konzentrierter Salzsäure zugegeben und die Fluissigkeit wurde auf die Hälfte eingedampft. Man wiederholte den Zusatz von $500 \mathrm{~cm}^{3}$ Salzsäure und engte dann auf ca. $250 \mathrm{~cm}^{3}$ ein. Als die Flüssigkeit auf $30^{\circ} \mathrm{C}$. abgekühlt war, fügte man die Lösung von $50 \mathrm{gr}$ Kaliumchlorid in $150 \mathrm{~cm}^{3}$ Wasser hinzu, wobei sofort das violettbraune Kalium-nitroso-pentachloro-rutheneat ausfiel.

1) Vergl. A. Werner, B. 40, 2620 (1907). 
Nach einer Viertelstunde wurde das Salz abgesaugt, mit gesättigter Kaliumchloridlösung und dann mit etwas Alkohol und ̈̈ther gewaschen. Das auf die Hälfte eingeengte Filtrat lieferte beim Erkalten noch ziemlich viel Salz, vermengt mit Kaliumchlorid. Die abgesaugte Krystallisation wurde vorsichtig mit kaltem Wasser auf dem Filter ausgelaugt, wobei alles Kaliumchlorid mit wenig Ruthenverbindung in Lösung ging; das zurückgebliebene Salz wurde wie die erste Fällung behandelt.

Die Gesamtausbeute an lufttrockenem Salz betrug 50 gr.

Darstellung des Nitroso-hydroxo-tetrammin-ruthenebromids

$$
\left[\begin{array}{l}
\mathrm{NO} \\
\mathrm{HO}
\end{array}\right.
$$

Das obige Kaliumsalz wurde in zwei Portionen wie folgt verarbeitet:

$25 \mathrm{gr}$ davon wurden in $150 \mathrm{~cm}^{3}$ Wasser von $30^{\circ} \mathrm{C}$. gelöst, filtriert und mit $50 \mathrm{~cm}^{3}$ ca. 18 proz. Ammoniak versetzt. Die Flüssigkeit wurde so lange auf dem Wasserbade erwärmt, bis ihre anfänglich rote Farbe einer gelbbraunen gewichen war. Man versetzte die abgekühlte Lösung in einer Schale mit $100 \mathrm{gr}$ pulverisiertem Natriumbromid, und förderte die Krystallisation durch Reiben der Wandung. Nach ca. 20 Minuten wurde der gebildete graugelbe Niederschlag abgesaugt und mit Alkohol und etwas Äther gewaschen. Ausbeute (lufttrocken) 12,5 gr. Die zweite Portion ergab 12,8 gr.

Darstellung der Nitroso-hydroxo-diäthylendiamin-ruthenereihe.

$$
\left[\begin{array}{lll}
\mathrm{NO} & \mathrm{Ru} & \mathrm{Cn}_{2} \\
\mathrm{HO} & \mathrm{O} & \mathrm{X}_{2}
\end{array}\right.
$$

20 gr Nitroso-hydroxo-tetrammin-ruthenebromid wurden in einem Kölbchen mit $80 \mathrm{~cm}^{3} 10$ proz. wässeriger Athylendiaminlösung übergossen und eine Stunde lang auf dem Wasserbade erhitzt. Das gelbe Salz ging dabei unter reichlicher Entwicklung von Ammoniak in Lösung. Nachdem die grösste Menge des entstandenen Ammoniaks verjagt war, wurde die gelbbraune Lösung filtriert und das abgekühlte Filtrat mit 25 gr pulverisiertem Kaliumjodid versetzt, wodurch rasch ein dicker Brei von glänzenden, gold-

1) Vergl. A. Werner, B. 40, 262 (1907). 
gelben Blättchen ausgeschieden wurde. Diese Fällung wurde abgesaugt, mit Alkohol und Äther gewaschen und an der Luft getrocknet; aus dem auf die Hälfte eingeengten Filtrat krystallisierte beim Erkalten noch eine beträchtliche Menge des gleichen, jedoch. weniger reinen Salzes, welche gleich der ersten behandelt wurde. Die Gesamtausbeute betrug $12 \mathrm{gr}$.

Dieses Rohjodid der neuen Reihe stellte kleine Blättchen von hellgelber Farbe mit einem graubraunen Stich dar, welcher wohl durch eine Spur kolloidal verteilten Rutheniums verursacht ist. Diese Verunreinigung lässt sich auch durch wiederholtes Umkrystallisieren des Salzes aus den sorgfältig filtrierten Lösungen nicht entfernen; das mehrmals umkrystallisierte Jodid lieferte folgende Analysendaten.

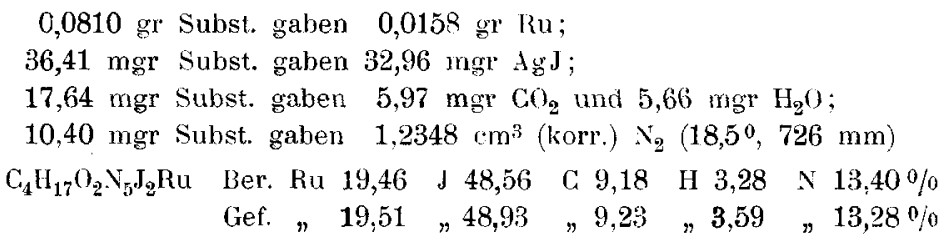

Diese Befunde lassen über die Zusammensetzung sowohl als über die Natur der Verunreinigung keinen Zweifel ibrig.

Salze der Nitroso-hydroxo-diäthylendiamin-ruthenereihe.

$$
\text { Jo did: }\left[\begin{array}{lll}
\mathrm{NO} & \mathrm{Nu} & \mathrm{HO} \\
\mathrm{HO}
\end{array}\right] \mathrm{J}_{2}
$$

Wie schon erwähnt, lässt sich das Rohjodid der Reihe durch Umkrystallisieren nicht reinigen; man gelangt indessen leicht auf einem Umwege zum Ziel. Lässt man nämlich in der Lösung des durch Rutheniumschwarz verunreinigten Jodides einen voluminösen, doch gut filtrierbaren Niederschlag entstehen, so wird die Verunreinigung mitgerissen und das Filtrat völlig frei davon.

Demgemäss wurde die konzentrierte Lösung von $10 \mathrm{gr}$ Rohjodid mit soviel 10 proz. Silbernitratlösung tropfenweise unter Umrühren versetzt, als zur Fällung ungefähr eines Viertels des gesamten Jods ausreichend war. Man erhitzte eine kurze Zeit auf dem Wasserbade, filtrierte das Silberjodid ab und fällte das klare, grünlichgelbe Filtrat nach genügendem Einengen mit pulverisiertem Kaliumjodid im Überschuss; das reine Jodid scheidet sich dabei in schön glänzenden, goldgelben Blättchen aus (lufttrocken 9,5 gr). 
Das Salz ist bei Zimmertemperatur ziemlich gut löslich in Wasser, leicht beim Erwärmen. Man erhält es beim Umkrystallisieren als kleine wetzsteinförmige Täfelchen. Es ist wasserfrei.

$$
\begin{aligned}
& 0,0883 \text { gr Subst. gaben } 0,0171 \text { gr Ru } \\
& 34,86 \text { mgr Subst. gaben } 31,28 \mathrm{mgr} \text { AgJ } \\
& \mathrm{C}_{4} \mathrm{H}_{17} \mathrm{O}_{2} \mathrm{~N}_{5} \mathrm{~J}_{2} \mathrm{Ru} \quad \text { Ber. Ru 19,46 J 48,56\% } \\
& \text { Gef. } \text {, } 19,37,48,50 \% \\
& \text { Bromid: }\left[\begin{array}{lll}
\mathrm{NO} & \text { Bu } & \text { en } \\
\mathrm{HO}
\end{array}\right] \mathrm{Br}_{2}
\end{aligned}
$$

$1 \mathrm{gr}$ reines Jodid wurde mit frisch gefälltem Silberbromid (aus $1 \mathrm{gr}$ Silbernitrat) und $10 \mathrm{~cm}^{3}$ Wasser angerührt und eine Stunde lang auf dem Wasserbade erhitzt. Die hierauf von den Silberhalogeniden abgesaugte Lösung wurde samt dem Waschwasser des Niederschlages auf ca. $5 \mathbf{~ c m}^{3}$ eingeengt und über konzentrierter Schwefelsäure in einem Exsikkator zur Krystallisation gestellt. Über Nacht bildeten sich schöne, gelbe, dünne Prismen (Nadeln). Das Bromid wurde abgesaugt und auf einer Tonplatte an der Luft getrocknet. Ausbeute 0,6 gr. Das Salz ist wasserfrei und leicht löslich in Wasser.

$$
\begin{aligned}
& 0,0810 \text { gr Subst. gaben } 0,0191 \mathrm{gr} \text { ku } \\
& 32,14 \text { mgr Subst. gaben } 28,13 \mathrm{mgr} \mathrm{AgBr} \\
& \mathrm{C}_{4} \mathrm{H}_{17} \mathrm{O}_{2} \mathrm{~N}_{5} \mathrm{Br} \mathrm{r}_{2} \mathrm{Ru} \text { Ber. Ru 23,72 Br } 37,28 \% \\
& \text { Gef. " } 23,58 \text { n } 37,24 \% \\
& \text { Chlorid: } \quad\left[\begin{array}{lll}
\mathrm{NO} & \mathrm{Ru} \in \mathrm{B}_{2}
\end{array}\right] \mathrm{Cl}_{2}
\end{aligned}
$$

1 gr reines Jodid wurde mit frisch gefälltem Silberchlorid aus 1 gr Silbernitrat in der gleichen Weise umgesetzt und auf Chlorid verarbeitet, wie bei der Darstellung des Bromids beschrieben ist. Es wurden 0,5 gr feine, gelbe Nadeln erhalten. Das leicht lösliche Chlorid enthält kein Krystallwasser.

0,0784 gr Subst. gaben $0,0234 \mathrm{gr} \mathrm{Ru}$

$30,26 \mathrm{mgr}$ Subst. gaben $25,45 \mathrm{mgr} \mathrm{AgCl}$

$$
\begin{aligned}
\mathrm{C}_{4} \mathrm{H}_{17} \mathrm{O}_{2} \mathrm{~N}_{5} \mathrm{Cl}_{2} \mathrm{Ru} & \text { Ber. Ru } 29,93 \text { Cl } 20,87 \% \\
& \text { Gef. } " 24,85 \text { " } 20,81 \% \\
\text { Nitrat: } & {\left[\begin{array}{l}
\mathrm{NO} \\
\mathrm{HO}
\end{array} \mathrm{Ru} \mathrm{en}_{2}\right]\left(\mathrm{NO}_{3}\right)_{2} }
\end{aligned}
$$

Man setzte 1,5 gr reines Jodid mit 0,975 gr Silbernitrat in $10 \mathrm{~cm}^{3}$ Wasser auf dem Wasserbade um, engte die vom Silber- 
jodid abgesaugte klare, gelbe Lösung bis auf ca. $6 \mathrm{~cm}^{3}$ ein und liess über konzentrierter Schwefelsäure stehen. Nach mehreren Stunden krystallisierte das Nitrat der Reihe in Form von schönen, derben, dunkelgelben Prismen aus. Das auf Ton an der Luft getrocknete Salz wog 0,6 gr. Das Nitrat ist leicht löslich und enthält kein Krystallwasser.

$$
\begin{aligned}
& 0,0826 \mathrm{gr} \text { Subst. gaben } 0,0212 \mathrm{gr} \text { ku } \\
& \text { 5,12 mgr Subst. gahen 1,1466 } \mathrm{cm}^{3} \text { (korr.) } \mathrm{N}_{2}\left(19^{0}, 724 \mathrm{~mm}\right) \\
& \mathrm{C}_{4} \mathrm{H}_{17} \mathrm{O}_{8} \mathrm{~N}_{7} \mathrm{Ru} \text { Ber. Ru 25,82 N 24,90\% } \\
& \text { Ger. " } 25,67,25,05 \% \\
& \text { Sulfat: }\left[\begin{array}{lll}
\mathrm{NO} & \mathrm{ku} & \mathrm{en} \\
\mathrm{HO}
\end{array}\right] \mathrm{SO}_{4}
\end{aligned}
$$

$1,2 \mathrm{gr}$ reines Jodid wurden mit $0,716 \mathrm{gr}$ Silbersulfat und $10 \mathrm{~cm}^{3}$ Wasser angerührt und eine Stunde lang auf dem Wasserbade erhitzt. Die vom Silberjodid abgesaugte Lösung wurde auf ca. $5 \mathrm{~cm}^{3}$ eingeengt und über konzentrierter Schwefelsäure stehen gelassen. Nach einigen Stunden krystallisierte das Sulfat in Form von gelben Nadeln aus. Das in üblicher Weise isolierte Salz wog lufttrocken 0,6 gr. Das Sulfat ist wasserfrei und leicht löslich in Wasser.

$$
\begin{aligned}
& 0,0745 \text { gr Sulsst. gaben } 0,0212 \text { su } \\
& 0,1218 \mathrm{gr} \text { Subst, gaben } 0,0543 \mathrm{gr} \mathrm{BaSO}_{4} \\
& \mathrm{C}_{4} \mathrm{H}_{17} \mathrm{O}_{6} \mathrm{~N}_{5} \mathrm{SRu} \text { Ber. Ru 28,52 } \mathrm{SO}_{4} 26,33 \% \\
& \text { Gef. } \quad 28,46 \text { "26,23\% } \\
& \text { Perchlorat: }\left[\begin{array}{lll}
\mathrm{ON} \\
\mathrm{HO}
\end{array} \mathrm{Ru}^{\mathrm{N}} \mathrm{H}_{2}\right]\left(\mathrm{ClO}_{4}\right)_{2}
\end{aligned}
$$

Dieses schöne Salz wurde aus dem weiter unten beschriebenen $\mathrm{d}$-( $\alpha$-Brom)- $\pi$-Camphersulfonat der Reihe bereitet. $3 \mathrm{gr}$ davon wurden mit 70 proz. Überchlorsäure gerade uiberschichtet, gut durchgerieben und mit $5 \mathrm{~cm}^{3}$ Alkohol verruihrt. Das gelbe Krystallmehl des Perchlorats wurde scharf abgesaugt und mit Alkohol und Äther säurefrei gewaschen. Das so erhaltene Salz wurde in $6 \mathrm{~cm}^{3}$ heissem Wasser gelöst und über Schwefelsäure stehen gelassen. Nach einigen Stunden wurden die auskrystallisierten grossen flachen Prismen (Tafeln) von schöner gelber Farbe in üblicher Weise isoliert $(0,8 \mathrm{gr})$. 
Das leicht lösliche Perchlorat enthält kein Krystallwasser.

0,0852 gr Subst. gaben $0,0184 \mathrm{gr} \mathrm{Ru}$

7,48 mgr Subst. gaben $1,0094 \mathrm{~cm}^{3}$ (korr.) $\mathrm{N}_{2}\left(19^{0}, 724 \mathrm{~mm}\right.$ )

$$
\begin{aligned}
& \mathrm{C}_{4} \mathrm{H}_{17} \mathrm{O}_{10} \mathrm{~N}_{5} \mathrm{Cl}_{2} \mathrm{Ru} \text { Ber. Ru } 21,74 \times 14,97 \% \\
& \text { Gef. , 21,60 , } 15,03 \% \\
& \text { d-( } \alpha \text {-Brom) }-\pi \text {-camphersulfonat: } \\
& {\left[\begin{array}{l}
O \mathrm{~N} \\
\mathrm{HO}
\end{array}\right.}
\end{aligned}
$$

Dieses Salz wurde zum Zweck der Spaltung des neuen Rutheniumkomplexes in optisch-aktive Komponenten dargestellt.

3 gr Rohjodid der Reihe wurden mit 4,996 gr d-( $\alpha$-brom)$\pi$-camphersulfonsaurem Silber und $20 \mathrm{~cm}^{3}$ Wasser so lange auf dem Wasserbade erhitzt, bis die gelbe liösung über dem zusammengeballten Silberjodid ganz klar erschien. Hierauf wurde sie vom Niederschlag abgesaugt, der letztere wurde mit ca. $5 \mathrm{~cm}^{3}$ Wasser nachgewaschen und die vereinigten Filtrate wurden auf ca. $10 \mathrm{~cm}^{3}$ eingeengt. Beim Stehen über konzentrierter Schwefelsäure erreichte die Lösung zuerst eine syrupöse Konsistenz, dann (nach 48 Stunden) krystallisierten derbe, gelbe, vielfach verwachsene Prismen aus. Nach dem Verdünnen der Mutterlauge mit etwas Alkohol wurden die Krystalle abgesaugt und auf Ton getrocknet, wobei sie rasch verwitterten. Im Exsikkator bis zur Gewichtskonstanz getrocknet, ergab das Salz folgende Analysenwerte:

$$
\begin{gathered}
0,2432 \text { gr Subst. gaben } 0,0275 \text { gr Ru } \\
\mathrm{C}_{24} \mathrm{H}_{45} \mathrm{O}_{10} \mathrm{~N}_{5} \mathrm{~S}_{2} \mathrm{Br}_{2} \mathrm{Ru} \text { Ber. Ru } 11,44 \%
\end{gathered}
$$

Eine kleine Probe des leicht löslichen Salzes wurde in wenig warmem Wasser gelöst und durch Zusatz von pulverisiertem Kaliumjodid in das entsprechende Jodid verwandelt; dieses schied sich dabei sofort in schön glänzenden Täfelchen vom Aussehen des Ausgangsjodids aus. Die Polarisation seiner wässerigen Lösung ergab in der Tat keine Drehung.

Das Salz wurde auf Perchlorat verarbeitet (s. d.).

$$
\text { d-Campher- } \alpha \text {-sulfonat: }\left[\begin{array}{l}
\mathrm{NO} \\
\mathrm{HO}
\end{array}\right.
$$

In Fortsetzung der Spaltungsversuche wurde $1 \mathrm{gr}$ Jodid der Reihe mit $1,3 \mathrm{gr}$ d-campher- $\alpha$-sulfonsaurem Silber in $6 \mathrm{~cm}^{3}$ 
Wasser auf dem Wasserbade umgesetzt. Nach dem Filtrieren wurde die klare Lösung samt dem Waschwasser des Silberjodidniederschlages bis auf ca. $3 \mathrm{~cm}^{3}$ eingeengt und über Schwefelsäure stehen gelassen. Nach 24 Stunden wurden die ausgeschiedenen Krystalle des d-Campher- $\alpha$-sulfonates abgesaugt und an der Luft getrocknet. Das leicht lösliche Salz stellt grosse gelbe Blätter dar und enthält kein Krystallwasser.

$$
\begin{aligned}
& 0,2185 \text { gr Subst. gaben } 0,0301 \text { gr } \mathrm{Ru} \\
& \mathrm{C}_{24} \mathrm{H}_{47} \mathrm{O}_{10} \mathrm{~N}_{5} \mathrm{~S}_{2} \mathrm{Ru} \text { Ber. Ru 13,91\% Gef. Hu 13,77\% }
\end{aligned}
$$

Als eine kleine Menge des Salzes in das entsprechende Jodid verwandelt wurde, erwies sich seine wässerige Lösung als optisch inaktiv.

Weitere Versuche zar spaltung der Reihe in optisch-aktive Komponenten.

a) Mittelst der d-Camphersäure.

$1 \mathrm{gr}$ Jodid wurde mit $6 \mathrm{~cm}^{3}$ Wasser und $1 \mathrm{gr}$ (ber. $0,8 \mathrm{gr}$ ) d-camphersaurem Silber angerïhrt unt so lange auf dem Wasserbade erhitzt, bis die Lösung über dem zusammengelıallten Gemisch ron gebildetem silberjodid und überschüssigem Silbercamphorat klar erschien. Die filtrierte Lösung samt Waschwasser wurde durch einen vorsichtigen Zusalz von verdünnter Salzsảure von ciner Spur Silber (als Camphorat in der Lïsung enthalten) befreit. Die hieranf filtrierte Lösung wurde auf ca. $3 \mathrm{~cm}^{3}$ eingeengt, wobei feine gelle Nädelchen krystallisierten, welche nach dem Erkalten die Flüssigkeit wattcähulich ausfüllten.

Das Salz hieit beim Absaugen so hartnäckig die Mutterlauge zurück, dass es zwischen Filtrierpapier abgepresst werden musste. Die so erhaltene gelbe IIasse zeigte kein deutliches krystallinisches Gefüge mehr; von einer Analysse wurde abgesehen.

Das aus dem Salz bereitete lodid erwies sich als optisch inaktiv.

b) Vittelst der (1-Weinsäure.

$1 \mathrm{gr}$ Jodid wurcie in $6 \mathrm{~cm}^{3}$ Wasser gelöst und auf dem Wasserbade nit 0,7 gr Silber-d-tartrat umgesetzt, bis die Lösung über dem Niederschlag klar erschien. Sie wurde abgesangt; den Niederschlag wusch man mit ca. $2 \mathrm{~cm}^{3}$ Wasser aus. Die vereinigten Filtrate wurden auf ca. $3 \mathrm{~cm}^{3}$ eingeengt und übes Schwefelsäure stehen gelassen. Die Lösung nahm allmählich eine syrupöse Konsistenz an; wach 48 Stunden krvstallisierten dicke, verwachsene, braungelbe Prismen aus. Man war gezwungen, die Mutterlauge mit etwas Alkohol zu rerdünnen, um die Krystalle absaugen zu können. Sie hielten imıner noch nicht unbeträchtliche Teile des dicken Syrups zurück und mussten durch Zerreiben auf einer 'Tonplatte davon befreit werden. Von einer Analyse des so erhattenen gelben Pulvers wurde abgesehen. 
Das entsprechende, aus diesem Salz durch Umsatz mit Kaliumjodid dargestellte fodid erwies sich in wässeriger Lösung ebenfalls als inaktir.

c) Mittelst d-Nitrocamphers.

Mit diesem spaltungsmittel konnten wir noch weniger erzielen. weil das entsprechende Salz nicht krystallisierte.

\section{Zusammenfassung.}

1. Es wird über die Bildung der Nitroso-hydroxo-diäthylendiamin-ruthenereihe:

$$
\left[\begin{array}{lll}
\text { NO } & \text { Ru } & \text { en: } \\
\text { Ho }
\end{array}\right] \mathrm{X}_{2}
$$

berichtet, welche den ersten Typus der Rutheniumkomplexe mit Aminen (Diaminen) darstellt.

2. Es werden einige Salze dieser Reihe beschrieben.

3. Es wird auf Grund der negativen Resultate der Spaltungsversuche der Reihe mit Hilfe von fünf verschiedenen optischaktiven organischen Säuren mit Vorbehalt auf die 1,6(trans-) Stellung der NO- und OH-Gruppen im Komplex geschlossen.

Zürich, Chemisches Laboratorium der Universität, 28. September 1920. 